\title{
METODOLOGÍA PARA LA IMPLEMENTACIÓN DE LA TECNOLOGÍA IDENTIFICACIÓN POR RADIOFRECUENCIA EN ENTORNOS INDUSTRIALES Y SANITARIOS EN COLOMBIA.
}

\author{
METHODOLOGY FOR IMPLEMENTING RADIO FREQUENCY IDENTIFICATION \\ TECHNOLOGY IN INDUSTRIAL AND HEALTH ENVIRONMENTS IN COLOMBIA.
}

Diana Isabel Trujillo Cifuentes ${ }^{1}$, Oscar J. Calderón C².

1. Universidad del Cauca - Pontificia Universidad Javeriana Cali. Popayán Colombia.ditrujillo@unicauca.edu.co

2. Universidad del Cauca. Popayán - Colombia. oscarc@unicauca.edu.co

Recibido: 07 de febrero de 2014

Aceptado: 25 de marzo de 2014

*Correspondencia del autor. . Email:

\section{RESUMEN}

La tecnología de Identificación por Radio Frecuencia (RFID, Radio Frequency Identification) es una de las opciones de identificación de productos más utilizadas en la actualidad, ya que permite mejorar los procesos relacionados con el manejo de información de los productos en las cadenas de abastecimiento y en entornos sanitarios. Sus características convierten a RFID en la mejor alternativa para procesos de identificación de productos, frente a otros sistemas existentes, como los códigos de barra. Sin embargo, acorde a la literatura consultada, se encuentran pocas referencias a metodologías que guíen al usuario en la forma de implementar esta tecnología en los entornos indicados. El presente artículo propone una metodología para la implementación de RFID, para lo cual se realiza un proceso de investigación en donde se analizan metodologías existentes, logrando finalmente plantear una metodología que aborda aspectos tecnológicos y gerenciales.

Palabras clave: Cadena de abastecimiento, entorno sanitario, Identificación por Radio Frecuencia (RFID), Metodología.

\begin{abstract}
The RFID (Radio Frequency Identification technology) is actually one of most used options to the identification of products because it allows to improve the process related with the management of product information in the supply chain and medical environments. Its features make RFID the best option in identification products process over other systems such as barcodes. However, according to the references consulted, there are few methodologies that guide the user on how to implement this technology in the environments indicated. The present article proposes a methodology for the implementation of RFID, for which we realize a research where existing methodologies are discussed to finally propose a methodology that addresses technological and managerial aspects.
\end{abstract}

Keywords: Supply Chain, Health Environment, Radio Frequency Identification (RFID), Methodology. 


\section{INTRODUCCIÓN}

El control de la cadena de abastecimiento en tiempo real es hoy el principal objetivo que tienen las diferentes compañías en el país (1). El manejo actual que se realiza de esta cadena mediante la tecnología del código de barras es ineficiente y tedioso (2). La Tecnología RFID permite optimizar los procesos de seguimiento y control de productos en las cadenas de abastecimiento, gracias al uso de sistemas radio que aseguran trazabilidad.

La tecnología RFID mediante el uso de elementos llamados etiquetas (Tags), que contienen la información detallada de cada producto, el uso de lectores y las ondas de radio, permite identificar productos sin necesidad de tener contacto directo con los mismos (3).

De la misma forma en que RFID se convierte en una herramienta potencial para las cadenas de abastecimiento, también lo hace para el campo de la salud en donde se utiliza para el control y supervisión de la información de pacientes e insumos médicos (4).

Sin embargo, acorde a la literatura consultada se encuentran pocas referencias que indiquen una metodología que guíe al usuario en la forma de implementar la tecnología RFID en contextos de salud e industriales. Debido a ello, el presente artículo se enfoca en el planteamiento de una metodología que sirva como referente para los empresarios que propendan por la adecuada implementación de la tecnología.

Tanto en la cadena de abastecimiento como en los entornos de salud, la implementación de RFID se debe hacer de una forma estratégica o metodológica, ello garantiza que realmente sea una alternativa que permita mayor eficiencia en sus procesos y obtener mejores resultados en los seguimientos de sus productos. Acoger una metodología permite organizar los tiempos del proyecto, disminuir los riesgos, hacer predecible el desarrollo, permite que los nuevos integrantes de una empresa aprendan de forma más rápida cómo hacer el trabajo, logra muy buenas curvas de aprendizaje, y lo fundamental es que permite lograr una apropiada sistematización de sus procesos. Sin embargo, la implementación de RFID en una organización requiere modificaciones en la cultura organizacional, ya que las costumbres y las formas de realizar los procesos, pueden constituir una barrera para cualquier situación que implique cambios en la organización
(5).Acorde al Instituto de Gestión de Proyectos (PMI, Project Management Institute), una metodología es una orientación en donde se dan "buenas prácticas" que el equipo de un proyecto puede tomar como referencia sin necesidad de seguir estrictamente lo indicado (6), ya que de acuerdo a la empresa en que se esté aplicando pueden requerirse modificaciones.

El artículo se divide en cuatro secciones, la sección II describe los conceptos teóricos de RFID, la sección III presenta la metodología y en la sección IV se muestran las conclusiones.

\section{TECNOLOGÍA RFID.}

\section{A. Marco teórico.}

Un sistema RFID está compuesto por cuatro componentes básicos: una etiqueta o tag (que tiene un número único de identificación), un lector, una antena, y un sistema de información o middleware (7). Las etiquetas pueden ser pasivas o activas: las pasivas son utilizadas para la identificación de pacientes o insumos, mientras que las activas son utilizadas para propósitos de seguimiento o control de trazabilidad. El funcionamiento de un sistema RFID inicia cuando el lector solicita la información a las etiquetas, aquellas que se encuentren dentro del rango de operación responden enviando la información solicitada. El lector envía la información al middleware quien analiza y almacena los datos, integrándolos con el sistema de información de la compañía (8).

La figura 1 muestra el esquema de operación de RFID.

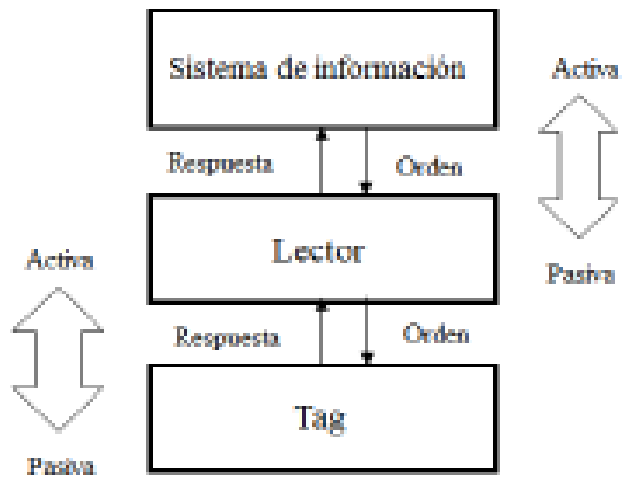

Figura 1. Esquema de operación de RFID

Tanto en los entornos sanitarios como industriales, los sistemas RFID pueden combinarse con otras tecnologías como Bluetooth, o sensores para diferentes propósitos.

\footnotetext{
1. Imágen adaptada de "Integration of RFID Technique and E-Commerce Logistics”. IEEE Computer Society, 152-155. Año 2009
} 


\section{B. Contextos de aplicación de la tecnología.}

RFID es una tecnología que puede ser utilizada en diferentes contextos o entornos, siendo significativos los siguientes (9):

Industria Logística: la cadena de abastecimiento compuesta por los proveedores, la producción, el almacenamiento y la distribución al cliente, mediante RFID pueden lograr eficiencia en sus procesos. Empresas de talla mundial como es el caso de Walmart lo confirman.

Industria de la salud: en el sector de la salud se destacan los procesos de control de insumos médicos, la seguridad en procedimientos médicos, el control y seguimiento de pacientes.

Industria de alimentos: en esta industria los bienes en su mayoría son perecederos, si no hay un manejo adecuado en la cadena de transporte la vida útil del producto se reduce teniendo pérdidas de productos. De acuerdo a la Administración de Drogas y Alimentos (FDA, Food and Drug Administration) de los Estados Unidos, aproximadamente el 20\% de los alimentos son descartados por deterioros en la cadena de suministro (9). RFID permite realizar seguimiento del estado de los alimentos en tiempo real reduciendo situaciones de deterioro de productos.

Industria de la moda: En el comercio de los minoristas de ropa, aplicaciones RFID permiten experiencias de compra más agradables y rápidas para el cliente, tal es el caso del espejo inteligente ubicado en los vestidores de los almacenes. El espejo es realmente un lector RFID, que identifica los productos que hay en stock permitiéndole visualizar a los usuarios diferentes opciones de compra de una manera ágil e interactiva.

Aplicaciones para viajes internacionales: la implementación de tags RFID en los pasaportes de los viajeros, es una gran ayuda que agilizaría múltiples procesos de documentación para el ingreso a un país.

\section{Beneficios que brinda la tecnología}

La tabla 1 resume los beneficios potenciales que trae RFID en entornos sanitarios e industriales.

\section{Limitaciones de RFID.}

A pesar de que RFID es una tecnología prometedora para la identificación de objetos y que tiene el potencial de brindar ventajas competitivas, tiene algunas limitaciones que deben mejorarse. La tabla 2 resume las limitaciones de RFID en entornos sanitarios e industriales.

Tabla 1. Beneficios de la aplicación de RFID.

\begin{tabular}{|c|c|c|}
\hline & $\begin{array}{c}\text { Entorno Sanitario [4] } \\
{[10]}\end{array}$ & $\begin{array}{c}\text { Entorno industrial } \\
{[11]}\end{array}$ \\
\hline 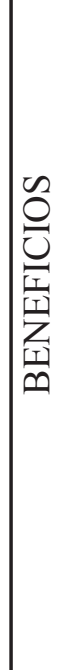 & 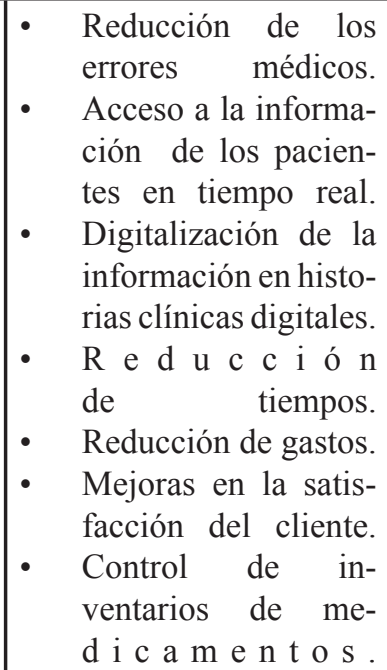 & $\begin{array}{l}\text { - Identificación rápi- } \\
\text { da de bienes. } \\
\text { Transmisión rápida } \\
\text { y precisa de la in- } \\
\text { formación. } \\
\text { - Trazabilidad en la } \\
\text { cadena. } \\
\text { Disminución de } \\
\text { la falsificación de } \\
\text { bienes. } \\
\text { Mejoras en la co- } \\
\text { municación entre } \\
\text { los eslabones de la } \\
\text { cadena. }\end{array}$ \\
\hline
\end{tabular}

\section{RESULTADOS Y DISCUSIÓN}

El control de cadenas de abastecimiento y el control de activos y pacientes, a pesar de darse en entornos muy diferentes, tienen un objetivo en común: lograr efectividad y eficiencia en cada una de las labores desarrolladas. Acorde a ello, teniendo en cuenta que se propone una metodología de carácter gerencial, es posible generalizarla, garantizándole al usuario una guía para la implementación de la tecnología.

\section{A. Metodología.}

El objetivo principal de la investigación es proponer una metodología que permita la implementación de un proyecto RFID en cadenas de abastecimiento y entornos sanitarios acorde a los requerimientos del cliente, en el tiempo indicado y con el presupuesto establecido. La metodología se propone teniendo en cuenta modelos utilizados en la administración de proyectos, en la construcción de software, en la implementación de proyectos tecnológicos que se considera aportan ideas significativas, como la Metodología para la administración de proyectos del PMI (6), metodologías utilizadas en CISCO (12), Metodología para la implementación de RFID en procesos de manufactura elaborada por el grupo BRIDGE (Building Radio frequency IDentification for the Global Envi- 
Tabla 2. Limitaciones de RFID.

\begin{tabular}{|c|c|}
\hline \multicolumn{2}{|c|}{ Limitaciones de RFID [4] } \\
\hline Limitación & Hallazgos \\
\hline Costos & $\begin{array}{l}\text { La infraestructura RFID es costosa } \\
\text { en comparación con la infraestruc- } \\
\text { tura de otro tipo de tecnologías. La } \\
\text { integración de los sistemas RFID } \\
\text { con los sistemas de información de } \\
\text { las compañías tiene un costo muy } \\
\text { elevado. }\end{array}$ \\
\hline Interferencias & $\begin{array}{l}\text { Los dispositivos que hay en las com- } \\
\text { pañías pueden fallar en presencia de } \\
\text { sistemas RFID, ello se ha probado } \\
\text { mediante test de interferencias. }\end{array}$ \\
\hline Ineficacia & $\begin{array}{l}\text { Una colocación incorrecta de las eti- } \\
\text { quetas causa que el sistema RFID no } \\
\text { funcione adecuadamente en la iden- } \\
\text { tificación de los objetos. La legibili- } \\
\text { dad de un tag se ve afectada en en- } \\
\text { tornos donde hay múltiples sistemas } \\
\text { RFID. }\end{array}$ \\
\hline Estandarización & $\begin{array}{l}\text { La falta de estandarización de los } \\
\text { protocolos para RFID causa intero- } \\
\text { perabilidad entre proveedores. }\end{array}$ \\
\hline $\begin{array}{l}\text { Privacidad y aspectos lega- } \\
\text { les. }\end{array}$ & $\begin{array}{l}\text { En los sistemas RFID, sea en entor- } \\
\text { nos industriales o sanitarios, infor- } \\
\text { mación confidencial queda expuesta, } \\
\text { las compañías deben garantizar la } \\
\text { ética en el manejo de la misma. }\end{array}$ \\
\hline
\end{tabular}

ronment) de la Unión Europea (13), y la Metodología para el desarrollo de aplicaciones RFID: un ejemplo práctico elaborada por Beatriz Pérez para optar por el título de Maestría en Tecnología de computo en el Instituto Politécnico Nacional de México (14).

La figura 2 ilustra la metodología propuesta, en donde se observan siete fases que cubren la totalidad de un proyecto de implementación de RFID: Análisis de Requerimientos, Planeación, Diseño, Implementación, Pruebas, Ajustes y Monitoreo, Cierre y una fase de documentación paralela a todas las fases.

\section{Análisis de requerimientos.}

En esta fase se tiene el primer acercamiento con el cliente, una adecuada comunicación permite determinar los requerimientos del proyecto. Acorde a la información obtenida se logra identificar el alcance del proyecto y el trabajo que se debe realizar, así como los riesgos que se pueden tener en la implementación. Las actividades que se deben realizar en esta fase son:

- Análisis del estado actual: conocer y analizar cómo se están llevando a cabo los procesos de la compañía, con el fin de identificar dónde RFID puede optimizar las tareas desarrolladas.

- Identificación de interesados: identificar y organizar los interesados de acuerdo a su nivel de afectación en la implementación del proyecto. Los interesados pueden ser: organizaciones o personas que participan de forma activa en el desarrollo del proyecto $\mathrm{u}$ organizaciones o personas cuyos intereses pueden verse afectados por la ejecución del proyecto.

- Determinación del alcance: indicar qué incluye el proyecto. En la definición del alcance son vitales los requerimientos del cliente, que pueden obte- 
nerse mediante procesos de documentación como entrevistas, talleres de opinión, encuestas, entre otros.

- Determinación de presupuesto: se debe conocer el presupuesto que se tiene para la implementación del proyecto.

- Identificar los recursos con los que cuenta la empresa ya sean recursos humanos e infraestructura que pueden servir para la implementación del proyecto.

- Estipular el grupo de trabajo.

\section{Planeación}

Después de haber identificado los requerimientos del cliente, es posible planear cómo se va a realizar la implementación del proyecto. En esta etapa es fundamental establecer las actividades que se van a realizar en la línea de tiempo que se tiene, de acuerdo al presupuesto que se ha establecido, las actividades deben incluir los planes de monitoreo del proyecto así como el plan de contingencias ante posibles riesgos que puedan presentarse. El resultado de esta fase es la programación de las actividades a realizar con el tiempo y los recursos requeridos, teniendo en cuenta rutas críticas.

Las actividades que se proponen en esta fase son:

- Análisis costo beneficio: Realizar una comparación entre los costos de la implementación de la tecnología y el ahorro que se generaría al integrar RFID a los procesos de la compañía.

- Establecimiento de la línea de tiempo: indicar las actividades a realizar mediante cronogramas.

- Establecer el plan de monitoreo: Definir las actividades mediante las cuales se controlará la ejecución del proyecto. Se debe establecer la frecuencia con que serán realizadas, así como los actores responsables.

- Definir los riesgos: Establecer un plan para cada uno de ellos. Se recomienda identificar el evento, la condición de riesgo, el umbral, la acción a tomar y la probabilidad de ocurrencia.

- Establecer los indicadores de desempeño del sistema: los cuales permitirán evaluar si el sistema implementado cumple con los objetivos del proyecto.

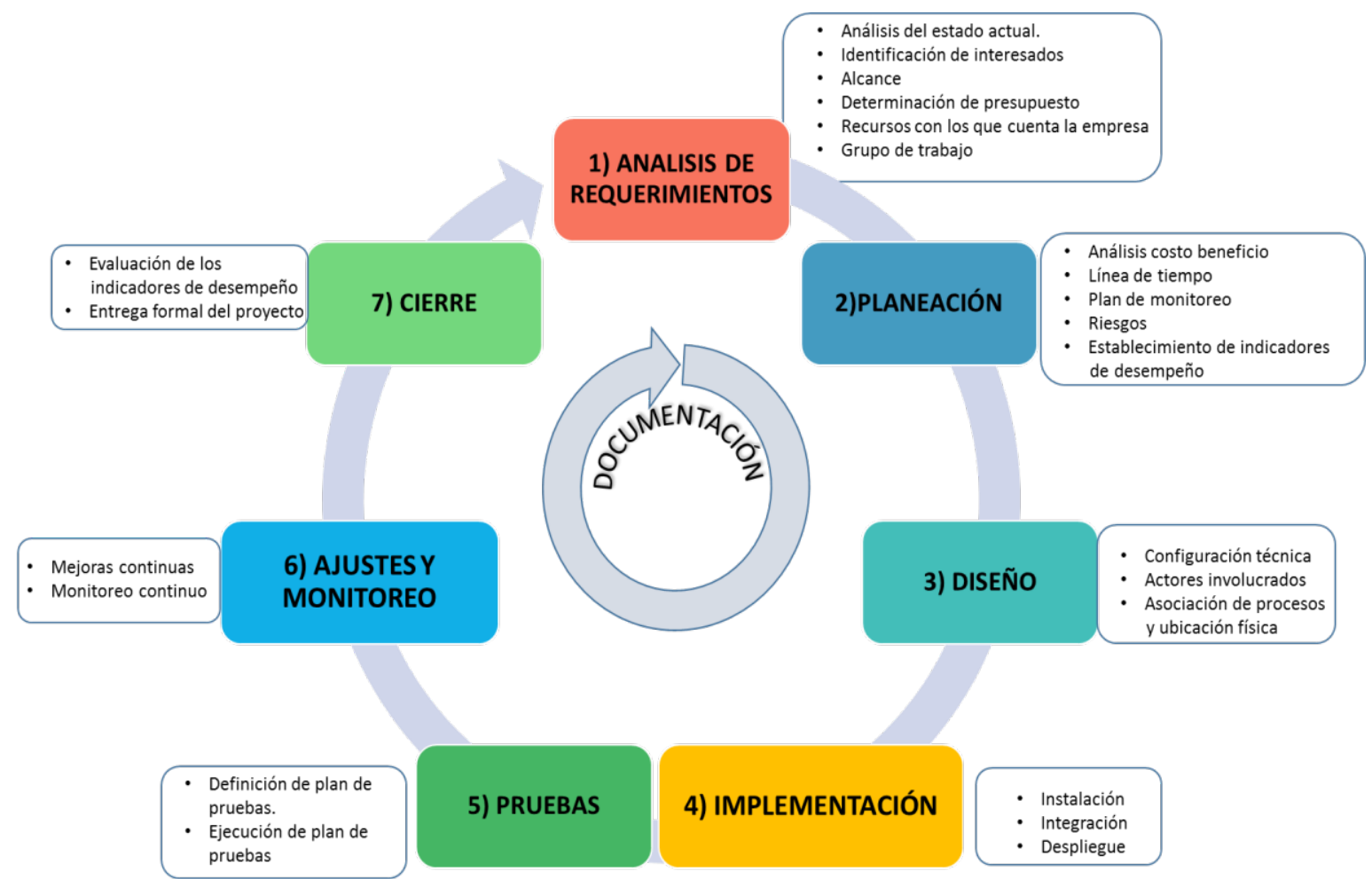

Figura 2. Metodología propuesta para la implementación de un proyecto RFID en entornos sanitarios e industriales. 


\section{Diseño}

De acuerdo a los requerimientos del cliente y teniendo en cuenta la planeación se procede a realizar el diseño del proyecto, ello incluye el diseño técnico de la solución y su integración con la infraestructura existente. El resultado de esta fase es una propuesta viable en términos de recursos humanos, financieros y tecnológicos que permita cumplir con el alcance del proyecto. Se proponen las siguientes actividades:

- Asociación de procesos con su ubicación física: Para poder realizar el diseño se deben asociar los procesos en donde se implementará RFID, con las ubicaciones físicas óptimas que permitan hacer más eficiente el desempeño del sistema RFID.

- Determinación de la configuración técnica: se debe establecer los equipos y la configuración adecuada de acuerdo a lo establecido en la fase de análisis de requerimientos.

- Actores involucrados: es necesario establecer los actores de cada proceso que desarrollarán tareas relacionadas con el funcionamiento del sistema RFID.

\section{Implementación}

Una vez se haya realizado el diseño se procede a la implementación de la solución. Es la fase más crítica ya que pone en ejecución lo que se ha planeado de manera teórica. Al finalizar esta etapa se tiene la implementación de la solución que se diseñó en la fase anterior, así como la integración al sistema de información de la compañía. Las actividades que se proponen son:

- Instalación: el primer paso es instalar todos los equipos de acuerdo a la configuración indicada en el diseño. Se hace la instalación de los tags en cada uno de los elementos, así como la ubicación de lectores, antenas y sistemas de información.

- Integración: Se procede a integrar el sistema RFID instalado con el sistema de información de la compañía.

- Despliegue: Finalmente se pone en funcionamiento la solución diseñada.

\section{Pruebas}

Una vez se haya implementado la solución diseñada de acuerdo a los requerimientos del cliente, es necesario realizar pruebas controladas del funcionamiento del sistema. Al final de esta fase se logra establecer si lo planeado teóricamente coincide con el funcionamiento físico del sistema. La fase de pruebas es de vital importancia ya que en caso de no coincidir as- pectos de la planeación teórica con el funcionamiento del sistema, se debe revisar y si es necesario se deben hacer los ajustes pertinentes. Se proponen las siguientes actividades:

- Definición de plan de pruebas

- Ejecución del plan de pruebas: para la ejecución del plan de pruebas se propone implementar una prueba piloto en un tiempo y espacio definido. Una prueba piloto permite probar la solución en un ambiente controlado logrando hacer ajustes, antes de llevar a cabo la ejecución real.

\section{Ajustes y monitoreo}

Después de realizar iteraciones de las pruebas de implementación, puede encontrarse necesario desarrollar ajustes en el sistema, cuidando que no se modifique el alcance establecido de acuerdo a los requerimientos del cliente. El monitoreo hace referencia al control o supervisión que se hace al sistema una vez se hayan llevado a cabo los ajustes necesarios, se debe monitorear de acuerdo al plan establecido que el sistema es operativo en el nivel de efectividad que se esperaba. La actividad principal en esta fase es:

- Mejoras continuas del sistema.

- Monitoreo continuo

\section{Cierre}

Como etapa final del proyecto, en el cierre se hace entrega formal del sistema operando de acuerdo a los requerimientos indicados en situaciones de operación real de la compañía, ya sea en la cadena de abastecimiento o en situaciones del entorno sanitario. Se considera que el proyecto se finaliza cuando se ha implementado y se ha evaluado el funcionamiento del sistema cumpliendo con lo indicado en la etapa de la planeación. Las actividades que se proponen en esta fase son:

- Evaluación de los indicadores de desempeño.

- Entrega formal del proyecto.

\section{Documentación}

El proceso de documentación se establece en la metodología como una fase paralela, es decir se realiza al tiempo que se va desarrollando cada una de las fases, no tendría sentido llegar al cierre del proyecto y empezar a organizar y obtener la información. Una buena documentación es un garante para el cliente en el momento de evaluar el funcionamiento global del proyecto, también constituye una ayuda fundamental en procesos de gestión de la calidad para la evaluación de indicadores de desempeño del sistema, es una 
herramienta de comunicación con niveles gerenciales que no están directamente inmersos en la ejecución del proyecto. La documentación debe realizarse de forma organizada y clara de tal forma que si hay un cambio de miembros del equipo, la transferencia del conocimiento del proyecto sea un proceso sencillo.

\section{CONCLUSIONES.}

La tecnología Identificación por Radio Frecuencia constituye una herramienta potencial para mejorar significativamente el desempeño de las cadenas de abastecimiento y para lograr sistemas de información hospitalarios seguros y eficientes. En Colombia, el uso de RFID es relativamente nuevo, son pocas las compañías que han optado por implementar esta tecnología, especialmente en el campo de la salud. Ello se debe a la ausencia de estudios previos que demuestren la viabilidad de usar RFID y el retorno de la inversión que genera, así como el desconocimiento de un procedimiento o una metodología guía que le permita a un determinado usuario abordar e implementar adecuadamente un proyecto de RFID, independiente del entorno en donde se encuentre. En el presente artículo se plantea una metodología que tiene siete fases estratégicas, que le permitirán al encargado del proyecto lograr de forma exitosa la ejecución de un proyecto de identificación por radio frecuencia, ya sea en un entorno sanitario o en un entorno industrial. Es posible generalizar la aplicación de esta metodología ya que los pasos indicados son de carácter gerencial o estratégico más que de carácter técnico.

\section{AGRADECIMIENTOS}

El presente artículo es el resultado del trabajo realizado en el proyecto "Aplicación de la tecnología RFID para la gestión logística en entornos industriales y sanitarios en Colombia", desarrollado en el marco de la Convocatoria Nacional 566, para Jóvenes Investigadores e Innovadores Año 2012 de Colciencias. Agradecimientos a la Universidad del Cauca y al Grupo de I+D GNTT de la Facultad de Ingeniería Electrónica y Telecomunicaciones por su apoyo al desarrollo del presente proyecto.

\section{BIBLIOGRAFÍA}

1. Vargas, L. "Supply Chain Management". Revista de Logística Legis, volumen 2013; (21),82- 88.

2. Morales, J. 2007. "La tecnología RFID en la cadena de suministro". AMECE GS1 México, Documentos 2007 030707,1-4. Consultado Enero 20, 2014, en http:// www.amece.org.mx/semanario/2007/docs/030707 RFID_en_Cadena_de_Suministro.pdf.

3. Jinrong, T. (2010), Haiquan, C. (2010). "Manufacturing logistics management using RFID: Dynamic and case study". International Conference on Computer Application and System Modeling, 2010; (12), 56-60.

4. Yao,W. , Chu, CH. (2010). y Li, Z. (2010). "The use of RFID in Healthcare: Benefits and Barriers". International Conference on RFID-Technology and Applications, 2010; 128-134.

5. Mendoza, M. "Metodología para la administración de proyectos: Una nueva cultura de trabajo". Innovaciones de Negocios, 2007; (4), 39-61.

6. Project Management Institute, A guide to the Project Management Body of Knowledge (PMBOK Guide). Fifth Edition. Newtown Square: Project Management Institute; 2013

7. Escobar de Lima,C.A., Vargas Ramírez, I.R., Quintero Acevedo, A. 2008. y López Acosta, A. 2008. "Diseño e implementación de un sistema hospitalario basado en tecnología RFID: Computación ágil, inteligente y móvil". Inteligencia y Tecnología Universidad Distrital, 2008; 22-37.

8. Pan, Y., Wang, Z. y Hu, Q. "Integration of RFID Technique and E-Commerce Logistics". IEEE Computer Society, 2009; 152-155.

9. Zhu, X., Samar, M. y Kurata, H. "A review of RFID technology and its managerial applications in different industries". Journal of Engineering and Technology Managenet, volumen 2012; (29), 152-167.

10. Tzeng, Sh. Chen, W. Pai, F. "Evaluating the bussines value of RFID: Evidence from five cases studies". Production Economics International Journal, volumen 2008; (112), 601-613.

11. Guo, Z., Zhang, X. "The Application of RFID Technology in the Logistics Supply Chain". IEEE Computer Society, 2010; 518-520.

12. Deployment Methodology. Suport CISCO site. Consultado Enero 29,2014, en http://www.cisco.com/c/en/ us/td/docs/voice_ip_comm/uc_system/UC7-0-1/system_description/SDmth.html

13. Brintrup, A. 2008. Report: Methodology for manufacturing process analysis for RFID implementation. Consultado Diciembre 20, 2013, en http://www.bridge-project.eu/data/File/BRIDGE_WP08_methodology_process_analysis.pdf

14. Pérez Bernabé, B. Metodología para el desarrollo de aplicaciones RFID: Un ejemplo práctico. [Tesis de Maestría]. México D.F.: Instituto Politécnico Nacional Centro de Innovación y desarrollo tecnológico en el cómputo del IPN; 2009. 